\title{
Preferencia de alimentos en adolescentes de 15 a 18 años de edad con menstruación regular en fase lútea en dos preparatorias del municipio de Aguascalientes
}

Martínez-Marín Karen Alejandra*, González-Briano Brenda Janeth*, Macías-Coronado Blanca Isela*, Monreal-Contreras Herma Selene*, Terán-Cruz María Vania*, Ibarra-Andrade Claudia Paulina*, Martín del Campo-Cervantes Judith**.

\section{Resumen}

- En esta investigación se buscó comparar la preferencia de ali-

- mentos en adolescentes de 15 a 18 años de edad con mens-

- truación regular en fase lútea de las preparatorias: CONALEP

- Aguascalientes plantel Il y Colegio Esperanza, del municipio de

- Aguascalientes. Se trata de un estudio descriptivo, longitudinal,

- observacional y prospectivo con una muestra de 44 adolescen-

- tes estudiantes de estas preparatorias. A quienes se les aplicó

- una frecuencia de alimentos en la fase lútea y fase no lútea

- de su menstruación para comparar su consumo de alimentos

- entre ambas fases. Como resultado se observó que existe un

- aumento en el consume de alimentos dulces en la fase lútea

- en comparación con la fase no lútea; los alimentos que mos-

- traron mayor aumento fueron mango, frappés, plátano, galletas

- dulces, brownie, dulces agridulces, jugos industrializados, hela-

- dos y té Arizona. Con lo anterior se concluye que la preferencia

- de alimentos dulces en estas adolescentes fue mayor en la

- fase lútea de su ciclo menstrual. LUX MÉDICA AÑ09, NÚMER028,

- SEPTIEMBRE-DICIEMBRE 2014 PP 47-51

\section{Abstract}

This research sought to compare the preference of food in teenagers from 15 to 18 years of age with regular menstruation in luteal phase of the high schools: CONALEP Aguascalientes campus II and Esperanza College, in Aguascalientes. This is a descriptive, longitudinal, observational, prospective study with a sample of 44 teenager's students of these schools, in who were administered food frequencies in the luteal phase and non-luteal phase of menstruation to compare their food consumption between two phases. As results it was observed that there is an increase in the consumption of sweet foods in the luteal phase compared with the non-luteal phase; the foods that showed greater increase were: mango, frappes, banana, cookies, brownies, sour candies, processed juices, ice cream and ice tea. It is concluded that the preference for sweet foods in these adolescents was highest in the luteal phase of their menstrual cycle. LUX MÉDICA AÑO 9, NÚMERO 28, SEPTIEMBREDICIEMBRE 2014 PP 47-51

Palabras clave: adolescentes, fase lútea, alimentos dulces

* Estudiantes de la Licenciatura en Nutrición del Centro de Ciencias de la Salud de la Universidad Autónoma de Aguascalientes.

** Profesora investigadora de la Licenciatura en Nutrición del Centro de Ciencias de la Salud de la Universidad Autónoma de Aguascalientes.

Fecha de recibido: 26 de febrero 2014

Fecha de aceptación: 23 de octubre 2014

Correspondencia: MCN Judith Martín del Campo Cervantes, Edificio 107, planta baja, Campus Universitario. Avenida Universidad \#940. Código posta 20131, Aguascalientes, Ags., México. Teléfono 9108436. Correo electrónico jmartic@correo.uaa.mx 


\section{Introducción}

Según la Organización Mundial de la Salud (OMS), el periodo de la adolescencia comprende de los 10 a los 19 años de edad. El desarrollo físico (cambios sexuales y corporales) que se produce durante la adolescencia va acompañado de importantes cambios psicosociales que caracterizan a este periodo como una etapa decisiva en el camino hacia la edad adulta ${ }^{1,2}$.

Entre estos cambios se encuentra el ciclo menstrual en las mujeres que se considera a partir del primer día del sangrado y consiste en cuatro etapas: la primera es la fase folicular, en la cual se desarrolla el folículo; la segunda es la fase peri-ovulatoria, donde el óvulo ya está maduro para la fecundación; la tercera es la fase pre-menstrual o también llamada fase lútea, aquí el endometrio se prepara para la implantación; la cuarta es la menstruación ${ }^{3-5}$.

Después de la fase de ovulación, inicia la fase lútea, es decir, cuando el folículo ovárico remanente se convierte en el cuerpo lúteo; es en esta fase donde se presentan cambios en las arteriolas del endometrio a causa de la progesterona como preparación para la fertilización $n^{3,6}$.

De acuerdo con Serret Montoya en la fase lútea se presenta un aumento en el consumo de energía que puede variar entre 80 y $500 \mathrm{kcal} /$ día, con un consumo elevado de hidratos de carbono y bajo en proteínas, además de cierta preferencia por los alimentos dulces lo que puede favorecer el estado de ánimo de la mujer y mejorar sus síntomas ${ }^{7-11}$. Los alimentos dulces son aquellos que al oxidarse en el calorímetro de bomba dan, en promedio, $4.15 \mathrm{kcal}$ por gramo de carbohidrato puro. Sin embargo, cabe destacar que los valores de combustible fisiológico de los alimentos cuando son oxidados por el cuerpo son un poco más bajos debido a la digestión incompleta de los nutrientes. Los experimentos realizados por Atwater en dietas mixtas típicas mostraron que la digestibilidad de los carbohidratos es de $98 \%{ }^{4}$.

\section{| | | | | | | | | | | | | | | | | | | | | | | | | | | | | | | | | | | | | | | | | | | | | | | | | | | | | | | | | | | | | | | | | | | | | | | | | | | | | | | | | | | | | | | | | | | | | | | | | | | | || ||}

\section{Material y métodos}

Diseño: Descriptivo, observacional, longitudinal, prospectivo.

Población: Adolescentes femeninas de 15 a 18 años de edad con ciclos menstruales regulares que acudan a:

* CONALEP Aguascalientes plantel II

* Colegio Esperanza

Muestra: 44 adolescentes con ciclo menstrual regular.

Muestreo: No probabilístico por cuota, seleccionando a 44 adolescentes de 15 a 18 años de edad de turno matutino, 22 adolescentes del Colegio Esperanza y 22 adolescentes del Conalep Aguascalientes plantel II.

El estudio se llevó a cabo en el periodo de agosto-octubre de 2013.

Material: 60 encuestas de selección de la muestra, 88 frecuencias de alimentos ${ }^{12}$. Se identificó a las adolescentes que cumplían con los criterios de inclusión y se les pidió que respondieran la frecuencia de alimentos, en la cual contes- 
taron la cantidad de veces a la semana que consumían alimentos de todos los grupos (frutas y verduras, cereales y alimentos de origen animal), esto con la finalidad de que no se dieran cuenta de que se evaluaría su consumo de alimentos dulces; y esta frecuencia de alimentos fue aplicada dos veces a cada adolescente seleccionada, la primera vez en su fase lútea y la segunda en su fase no lútea para comparar su consumo de alimentos dulces entre ambos periodos. Después de la recolección de datos se elaboró una base de datos y se realizó estadística descriptiva con el programa Excel.

\section{Criterios de Selección}

Criterios de inclusión: Mujer de 15 a 18 años de edad, presentar ciclos menstruales regulares de 25 a 40 días, no se encuentren embarazadas, no presenten enfermedades ginecológicas como síndrome de ovario poliquístico, fibromas, adherencias pélvicas, miomas uterinos, endometriosis; que estudien en Colegio Esperanza y CONALEP Aguascalientes plantel II

\section{|| || || |||||||||||||||||||||||||||||||||||||||||||||||||||||||||||||||||||||||||||||||||||||||||||||||||||||||||||||||||||||}

\section{Resultados}

En el análisis de cuantas comidas realizan al día se observó que la moda y la mediana fue de 3 comidas al día en todas las participantes; observando que el $72.73 \%$ señaló que se preocupa por su alimentación.

De la frecuencia de alimentos que se les aplicó a las adolescentes se obtuvieron los siguientes resultados (tabla I):

\section{Tabla I}

Incremento en el consumo de alimentos dulces en la fase lútea con respecto a la no lútea

\begin{tabular}{|lc|}
\hline ALIMENTOS DULCES & PORCENTAJE DE INCREMENTO \\
\hline Mango & $18.19 \%$ \\
\hline Frappes & $15.92 \%$ \\
\hline Plátano & $15.91 \%$ \\
\hline Galletas dulces & $15.91 \%$ \\
\hline Brownie & $14.45 \%$ \\
\hline Dulces agridulces & $13.64 \%$ \\
\hline Jugos industrializados & $13.63 \%$ \\
\hline Helados & $11.36 \%$ \\
\hline Té Arizona & $11.36 \%$ \\
\hline Gomitas & $11.32 \%$ \\
\hline Refrescos & $9.09 \%$ \\
\hline Churros azucarados o rellenos & $9.09 \%$ \\
\hline Bombones & $9.09 \%$ \\
\hline Paletas heladas & $9.08 \%$ \\
\hline Agua de fruta o de sabor & $6.83 \%$ \\
\hline Chocolate & $6.82 \%$ \\
\hline Caramelos dulces o macizos & $6.82 \%$ \\
\hline
\end{tabular}

$n=44$ adolescentes 


\section{Discusión}

Se investigó la relación entre la preferencia de alimentos de las adolescentes y la fase lútea del ciclo menstrual, debido a que se deseaba conocer cuáles eran los alimentos que consumían con mayor frecuencia en esta fase ya que, según Serret Montoya, ${ }^{6,7}$ en la fase lútea se presenta un aumento en el consumo de energía que puede variar entre 80 y $500 \mathrm{kcal} /$ día, con un consumo elevado de hidratos de carbono, además de indicar cierta sensibilidad por los alimentos dulces. Además, según Rosello $M .{ }^{3}$ en la etapa premenstrual existe una mayor preferencia por los hidratos de carbono; lo anterior se confirma con nuestra investigación puesto que se observó que en varios alimentos altos hubo un incremento en su consumo en la fase lútea en relación con la fase no lútea, como por ejemplo los pastelitos dulces y las galletas.

Rosello $M$ también refiere que en la fase lútea las características más frecuentes son la sed exagerada, incremento en el apetito y gusto exagerado por los dulces; en nuestro estudio esto se confirmó al observar que el consumo de bebidas dulces en la fase lútea incrementó, por ejemplo un aumento en el consumo de jugos industrializados con un $13.63 \%$ y los frappés un $15.92 \%$, ambos en el consumo de 1 a 6 veces por semana; mientras que el té helado mostró un aumento del $11.36 \%$ en el consumo de 2 a 6 veces por semana.

\section{Conclusiones}

Por todo lo anterior podemos decir que en la muestra estudiada, la preferencia de alimentos dulces en adolescentes de 15 a 18 años de edad fue mayor en la fase lútea de su ciclo menstrual (11.67\%).

Los resultados pueden desarrollar otras investigaciones, tales como un posible aumento de peso o aumento en el cólico menstrual.

\section{Bibliografía}

1. Organización Mundial de la Salud. Salud de la madre, el recién nacido, del niño y del adolescente. 2013. [citado 2013 Marzo 14] Disponible en: http://www.who.int/maternal_child_adolescent/topics/child/development/es/

2. Redondo C., Galdo G., García M. Atención al Adolescente. Santander Publican C. Ediciones de la Universidad de Cantaría. España. 2008: 3-4

3. Rosello M., Morales F., Pimentel D., Abadi-Levy T., Bustos H., Casanueva E. Ciclo menstrual y alimentación. Perinatol reprod. Hum. México. Abril Junio 2003; 2S (17): Pp: 61S-71S. [citado 2013 Marzo 14]; Disponible en:

http://www.mediagraphic.com/pdfs/inper/ip2003/ip032b.pdf

4. Gaete V Ximena, Codner D Ethel. Adelanto de la pubertad en Chile y el mundo. Rev. chil. pediatr. [Re- vista en la Internet]. 2008 Oct [citado 2013 Marzo 14]; 77(5): 456-465. Disponible en: http://www. scielo.cl/scielo.php?script=sci_arttext\&pid $=\mathrm{S} 03704$ $1062006000500002 \& \operatorname{lng}=$ es.

5. Zubirán S. Cuadernos de Nutrición. Alteraciones sexuales y reproductivas en las mujeres con obesidad. Fomento de Nutrición y Salud, A.C. Julio -Agosto 2009; 4(32): 138-140.

6. Serret Montoya Juana, Hernández Cabezza Abigail, Mendoza Rojas Ofelia, Cárdenas Navarrete Rocío. Alteraciones menstruales en adolescentes. Bol. Med. Hosp. Infant. Mex. [Revista en la Internet]. 2012 Feb [citado 2013 Marzo 14]; 69(1): 63-76. Disponible en: http://www.scielo.org $\mathrm{mx} / \mathrm{scielo}$.php? script $=$ sci_arttext\&pid $=\mathrm{S} 1665$ 11462012000100010\&lng $=$ es.

7. Serret M., Hernández C., Mendoza R., Cárdenas N. Alteraciones menstruales en adolescentes. Boletín médico hospital infantil México. [Revista en la Inter- 
net]. 2012 Feb 69(1): 63 -76. [citado 2013 Marzo 14]; Disponible en:

http://www.scielo.org.mx/scielo.php?script $=$ sci arttext\&pid=S1665-11462012000100010\&Ing=es.

8. Comité Nacional de Endocrinología. Trastornos del ciclo menstrual en la adolescencia. Arch. Argent. Pediatr. Buenos Aires jul. /ago. 2010; 108(4):363369 http://www.scielo.org.ar/pdf/aap/v108n4/ v108n4a18.pdf

9. Moore KL, Persaud TVN. Embriológía Clínica. Editorial ELSEVIER Saunders. 8va Edición. España 2009: 24-33
10. Alves H. SAMPAIO. Aspectos Nutricionales relacionados con el Ciclo Menstrual. Rev. Nutr., Campinas, 2002, 15S(3): 309S-17S

11. Ishii $C$, Nishino L, Herrerías de Campos CA. Caracterización vestibular en el ciclo menstrual. Revista Brasileira de Otorrinolaringología. Mayo/Junio ??2009; (Impr.) 3(75)

12. Monsalve J. M., González L I. Diseño de un cuestionario de frecuencia para evaluar ingesta alimentaria en la Universidad de Antioquia, Colombia. Nutrición Hospitalaria. Antioquia, Colombia 2011; 26(6):1337-134 